\title{
Prevención de tuberculosis en la edad escolar
}

\section{Sr. Editor:}

La tuberculosis (TB) es una enfermedad bacteriana crónica e infectocontagiosa producida por el Mycobacterium tuberculosis ${ }^{1}$, que sigue siendo una amenaza para la salud y el bienestar de la población mundial. Según informes de la Organización Mundial de la Salud (OMS), en el año 2011, 8,7 millones de personas enfermaron de tuberculosis y 1,4 millones murieron por causa de ésta, siendo más del $95 \%$ de las muertes en países de ingresos bajos y medianos; consolidándose como la segunda causa de mortalidad a nivel mundial, después del SIDA ${ }^{2}$. En Cuba, en el 2008, la incidencia de TB en todas sus formas notificadas era de 6,9 por 100.000 habitantes, aunque en el 2009 se notificaron 75 casos de TB en reclusos penitenciarios $(11,3 \%$ de un total de 666 casos). En la provincia de Santiago de Cuba, para este mismo año, se diagnosticaron 73 nuevos casos, pero aun así desde el año 2000 hasta el 2007, la incidencia de esta enfermedad en dicha comunidad tuvo una tendencia ascendente, aunque las recaídas aumentaron y exhibieron sus mayores porcentajes en los años 2005-2007 ${ }^{3}$.

La estrategia "Alto a la Tuberculosis", dirigida por la OMS, consiste en reducir radicalmente la carga mundial de TB para el año 2015. Dentro de los seis puntos que formula este plan, está el de "facultar a los afectados por la tuberculosis y a las comunidades", mediante la participación de estos en la prevención y la asistencia a la TB ${ }^{4}$.

La comunidad de "La Granjita", perteneciente al área de Chivirico, municipio de Guama, cercana a Santiago de Cuba- Cuba, ha reportado antecedentes de TB en los últimos años durante las pesquisas realizadas, es por eso que se motivó a la capacitación en promoción y prevención de la TB, con el objetivo de generar conciencia sobre TB en niños en edad escolar de la Escuela Primaria "Jesús Méndez" de esta comunidad.

Se realizó un estudio de intervención, sobre una muestra de 40 escolares ( 32 niñas 6 niños) de quinto grado de la Escuela Primaria, entre los 9 y 11 años de edad. Estos participantes fueron seleccionados mediante muestreo por conglomerados a partir de una población inicial de 60 alumnos matriculados. La intervención educativa consistió en tres etapas: una etapa diagnostica, seguida por las actividades de intervención educativa en promoción y prevención de TB, y una etapa final de evaluación.

En la primera etapa de la intervención, se observó que más del $70 \%$ de los participantes presentaron conocimientos inadecuados e incluso desconocimiento de TB en general. La segunda etapa de la intervención, consistió en seis sesiones con diferentes actividades dinámicas, con el fin de despertar la atención y confianza entre los participantes. En cada actividad se evaluaron conceptos básicos, vías de transmisión, métodos de prevención y grupos de riesgo de dicha enfermedad. Por último, en la tercera etapa, todos los participantes fueron evaluados nuevamente. El $100 \%$ de los encuestados respondieron adecuadamente a las siguientes preguntas: ¿Qué entiendes por tuberculosis?, ¿cómo se transmite la TB?, ¿cómo puedes prevenir la infección por TB?, ¿es posible evitar contagiarse por TB? y ¿qué tipo de personas tienen más riesgo de sufrir TB?

Generalmente el padecimiento de algunas enfermedades se debe a la falta de conocimiento y capacitación de la población; en los resultados obtenidos después de la intervención educativa realizada en la Escuela Primaria "Jesús Méndez" se demuestra que hubo aceptación de las actividades por parte de los estudiantes que participaron, así como también la adquisición y modificación de los conocimientos que tenían acerca de la TB. Aunque la población de este país de manera general presenta un alto nivel educacional, debido a los esfuerzos del gobierno por la educación del pueblo, el fortalecimiento de la movilización social y comunitaria y el desarrollo de recursos humanos académicos para la prevención de la TB no es suficiente en comunidades susceptibles a esta enfermedad. Por ello, es preciso seguir produciendo y difundiendo mensajes educativos acertados, potencialmente penetrantes y motivadores para los diferentes grupos de población, desde la edad escolar hasta la población geriátrica. También, continuar con una estrategia sostenible contra la TB es esencial para motivar al personal de los servicios de salud y promover su actualización científico-técnica, contribuyendo así a optimizar la prevención y el tratamiento de esta enfermedad con vistas a su eventual eliminación e implica, además, la creación de una alianza entre el sector de la salud y la comunidad: la población local, especialmente la más pobre y los pacientes con 
TB. Esto ayuda a formar promotores en salud, que potencien su papel protagonista en la comunidad, motivando y concienciando a los integrantes de su población sobre lo vulnerables pueden ser a la adquisición de esta infección y la posible prevención y el diagnostico oportuno de esta; ya que los esfuerzos aislados, lejos de ayudar, pueden incluso obstaculizar el camino a recorrer.

\section{BIBLIOGRAFÍA}

1. Ernst JD. The immunological life cycle of tuberculosis. Nat Rev Immunol. 2012;12(8):581-91.

2. Lienhardt C, Glaziou P, Uplekar M, Lonnroth K, Getahun $\mathrm{H}$, Raviglione M. Global tuberculosis control: lessons learnt and future prospects. Nat Rev Microbiol. 2012;10(6):40716.

3. González Díaz A, Perez Soler K, Sanchez Valdes L, Matthys F, Gonzalez Ochoa E, Van der Stuyft P. Tuberculosis incidence strata in Cuban municipalities: 1999-2002 and 2003-2006. Rev Panam Salud Publica. 2010;28(4):275-81.
4. González E, Risco GE, Borroto S, Perna A, Armas L. Tuberculosis mortality trends in cuba, 1998 to 2007. MEDICC review. 2009;11(1):42-7.

Miguel A. Bordó Pineda', Jessica Lozano Pérez $^{2}$, Luis Fang ${ }^{3}$, Antonio Díaz Caballero ${ }^{4}$

${ }^{1}$ Licenciado en Enfermería. Diplomado en Oncología General y Promoción en Salud. Miembro numerario de la Sociedad Cubana de Enfermería

${ }^{2}$ Estudiante de Odontología, Universidad de Cartagena. Grupo de investigaciones GITOUC.

${ }^{3}$ Odontólogo, MSc Inmunologia, Universidad de Cartagena.

${ }^{4}$ Odontólogo, Universidad de Cartagena. Especialista en Periodoncia por la Universidad Javeriana. Magíster en Educación. Director del grupo de investigaciones GITOUC.

Luis Fang Mercado. Facultad de Odontología, Universidad de Cartagena. Correo electrónico: luifang87@gmail.com

\section{Estrés académico y funcionalidad familiar en estudiantes de Odontología}

\section{Sr. Editor:}

Los programas de formación relacionados con la atención a pacientes, generan un alto nivel de estrés en los alumnos. Algunos estudios en estudiantes de medicina en Arabia Saudita ${ }^{1}$, enfermería en Brasil $^{2,3}$ y de psicología en Colombia ${ }^{4}$ reflejan que un alto nivel de estrés puede generar un efecto negativo en el funcionamiento cognoscitivo y en el aprendizaje ${ }^{3}$, y sugieren que la salud mental de los alumnos se deteriora después del comienzo de sus estudios y siguen siendo pobre durante todo el periodo 5 .

En los estudiantes de la Facultad de Odontología de la Universidad de Cartagena de Indias (Colombia) se manifiesta un abandono progresivo de los cuidados hacia su salud, para dar paso a otras prioridades y obligaciones académicas acompañadas de altos niveles de estrés, además, muchos viven situaciones estresantes a nivel familiar que dificultan la concentración y creación de buenos hábitos de estudio.

Barraza et $\mathrm{al}^{6}$ construye el modelo sistémicocognoscitivista del estrés académico y propone el uso del Inventario SISCO del Estrés Académico para su evaluación. Este inventario es autodescriptivo y se considera como un autoinforme que cumplimenta un sujeto sobre sí mismo y sobre distintos aspectos de su funcionamiento en situaciones académicas.

Al ser utilizado este instrumento en los estudiantes de Odontología, se encontró que las evaluaciones de los profesores y el tiempo limitado para cumplir sus actividades académicas, eran las situaciones que generaban más estrés académico. Las reacciones físicas más frecuentes, evaluadas por el mismo instrumento, eran los trastornos en el sueño y la somnolencia, seguida de los dolores de cabeza o migrañas, fatiga crónica y problemas de digestión. En cuanto a las reacciones psicológicas, las más observadas fueron la incapacidad para relajarse, seguido de la falta de concentración, la ansiedad y angustia.

El contexto social, cultural y familiar del estudiante puede influir en su adecuado rendimiento académico, siendo la familia una red de apoyo para ser utilizada como estrategia de afrontamiento del estrés académico ${ }^{7}$. Por ello la importancia de utilizar la funcionalidad familiar como un recurso para hacer predicciones en este sentido.

La mayoría de estudiantes que padecían de estrés académico y familias disfuncionales, reflejaron este problema bajo sentimientos de agresividad o aumento de irritabilidad, aumento o reducción 
del consumo de alimentos, y se comprometían las estrategias de afrontamiento del estrés según el Inventario SISCO. Estas situaciones pueden ocasionar un bajo rendimiento académico ${ }^{8}$ y una futura deserción entre los universitarios ${ }^{9}$.

Consideramos que es importante que las dependencias relacionadas con el bienestar de los universitarios en las instituciones educativas trabajen en la detección y control de riesgos presentes en los estudiantes a nivel familiar, social y comunitario que puedan comprometer su rendimiento académico, y promuevan el desarrollo adecuado de los proyectos de vida de los estudiantes universitarios.

\section{Bibliografía}

1. Abdulghani HM, AlKanhal AA, Mahmoud ES, Ponnamperuma GG, Alfaris EA. Stress and its effects on medical students: a cross-sectional study at a college of medicine in Saudi Arabia. J Health Popul Nutr. 2011; 29(5):516-22.

2. Braga PC, Souza LA, Evangelista RA, Pereira LV. The occurrence of headaches and their effect upon nursing undergraduate students Rev. esc. enferm. USP 2012; 46 (1):138-44

3. Dahlin M, Joneborg N, Runeson B. Stress and depression among medical students: a cross-sectional study. Med Educ. 2005; 39: 594-604.

4. Páez ML, Castaño JJ. Estilos de vida y salud en estudiantes de una Facultad de Psicología desde el Caribe 2010; 25: 155-178.

5. Rosal MC, Ockene IS, Ockene JK, Barrett SV, Ma Y, Hebert JR. A longitudinal study of students' depression at one medical college. Acad Med. 1997; 72:542-6.

6. Arrieta K, Díaz S, González F. Consumo de alcohol y problemas asociados en estudiantes de una universidad pública de Cartagena. Rev. Colomb. Psiquiat 2011; 40 (2): 215-228.

7. Bernal I. La Familia en la Determinación de la Salud. Rev Cubana Salud Pública 2003; 29(1):48-51.

8. Hernández M, Coronado O, Araujo V, Cerezo S. Desempeño académico de universitarios en relación con ansiedad escolar y auto-evaluación. Acta colombiana de psicología 2008; 11 (1): 13-23.

9. Contreras K, Caballero C, Palacio J, Pérez AM. Factores asociados al fracaso académico en estudiantes universitarios de Barranquilla (Colombia) Psicología desde el Caribe, 2008; 22: 110-135.

\section{Shyrley Díaz Cárdenas', Katherine Arrieta Vergara, ${ }^{2}$ Farith González Martinez ${ }^{3}$}

${ }^{1}$ Odontóloga Magíster en Salud Pública. Especialista en Salud Familiar y Gerencia en Salud.

${ }^{2}$ OD, Magíster en Salud Pública. Especialista en Gerencia en Salud.

${ }^{3} \mathrm{OD}$, Magister en Salud Pública, Especialista en Investigación Social

Facultad de Odontología, Departamento de Odontología Preventiva y Social. Universidad de Cartagena, Cartagena de Indias, Colombia. Correo electrónico: shyrley77@hotmail.com 